\title{
Los Estudios de Matemáticas en la Universidad de Alcalá en Tiempos de Carlos III y sus Precedentes
}

\author{
Studies of Mathematics at the University of Alcalá in the Era of Carlos III \\ and its Precedents
}

Javier Peralta*

\begin{abstract}
Resumen
A lo largo del siglo XVII, los estudios de matemáticas en las universidades españolas habían llegado casi a desaparecer, y esa situación se prolongó en parte del siglo XVIII, como se verá. El objetivo principal de este artículo es averiguar si el espíritu reformista del reinado de Carlos III tuvo incidencia en la enseñanza de las matemáticas en la Universidad de Alcalá. Para ello se analizan las reformas y planes de estudio que se establecieron y se examinan los libros de matemáticas recomendados. Se concluye que, efectivamente, la presencia de las matemáticas se incrementó, con la inclusión de dos asignaturas de matemáticas en la Facultad de Artes, y el nivel de sus contenidos evolucionó positivamente, incorporando a sus estudios la matemática moderna europea que había surgido con la Revolución Científica.
\end{abstract}

Palabras-clave: Universidad de Alcalá. Enseñanza de las Matemáticas. Carlos III. Reformismo. Siglos XVIXVIII.

\begin{abstract}
In the course of the seventeenth century, the studies of mathematics in Spanish universities had almost disappeared, and this situation continued in part of the eighteenth century, as will be seen here. The main purpose of this paper is to find whether the reforming spirit from the reign of Carlos III had an impact on the teaching of mathematics at the University of Alcalá. For this purpose, the reforms and syllabus that were established were analyzed and the books of mathematics recommended were examined. It is concluded that, indeed, the presence of mathematics increased, with the inclusion of two courses of mathematics in the Faculty of Arts, and the level of their contents evolved positively, incorporating to its studies the modern European mathematics that had emerged within the Scientific Revolution.
\end{abstract}

Keywords: University of Alcalá. Mathematics Teaching. Carlos III. Reformism, $16^{\text {th }}-18^{\text {th }}$ Centuries.

\section{Introducción}

A finales del siglo XII y principios del siguiente, se inicia un despertar científico en Europa, en el que juega un papel importante la creación de las universidades. Desde su fundación, las universidades organizan sus estudios en varias ramas del saber que se enseñan

\footnotetext{
" Licenciado y Doctor en Matemáticas por la Universidad Complutense de Madrid (UCM). Profesor Titular de Universidad de Matemática Aplicada, Universidad Autónoma de Madrid (UAM), Madrid, España. Dirección postal: Facultad de Formación de Profesorado y Educación, Ciudad Universitaria de Cantoblanco, 28049, Madrid, España. E-mail: javier.peralta@uam.es
} 
en cuatro o cinco Facultades, según la facultad o permiso recibido por el papa o el rey para impartir determinados conocimientos. Son las Facultades mayores: de Teología, Leyes, Cánones y Medicina; y una Facultad menor o de Artes.

La universidad española más antigua es la de Palencia, creada en 1208 por Alfonso VIII. Las más importantes, siguientes a ella y anteriores al siglo XVI, son, por orden de relevancia en aquella época, las de Salamanca (1215), Alcalá (1499) y Valladolid (1346): las llamadas universidades mayores ${ }^{1}$.

Las matemáticas en España no participan, sin embargo, del ambiente de progreso al que nos hemos referido. Hasta mediado del siglo XVI su enseñanza en nuestras universidades es aceptable, pero luego inician su declive, que continúa en el XVII.

El siglo XVIII, como es sabido, es el siglo de la Ilustración, dominado por la razón. Se produce una profunda evolución ideológica y se liberan las ataduras de las creencias tradicionales (es la era de la crítica, según Kant). En España, no obstante, no tiene la importancia que en otros países de su entorno, debido, en buena parte, a una mayor influencia de la religión. El espíritu de reforma se inicia con los primeros Borbones: Felipe $\mathrm{V}$ y Fernando VI; aunque a mediados del XVIII nos encontramos aún alejados de las verdaderas ideas renovadoras que imperan en Francia y otras naciones europeas. Con todo, ese prereformismo borbónico prepara el terreno al impulso que tendrá lugar durante el reinado de Carlos III (1759-1788), período principal de la Ilustración en España. ¿Y cómo afectarán al estudio de las matemáticas en la universidad de Alcalá las ideas renovadoras de Carlos III?

\section{Planteamiento y diseño de la investigación}

Los trabajos de historia de la educación española se ocupan de ese asunto, pero de manera general, no circunscrito a las matemáticas y, si tratan de enseñanza universitaria, suelen poner el énfasis en la Universidad de Salamanca, la más antigua de las ahora existentes $\mathrm{y}$, como se ha indicado, entonces la principal. Y lo mismo sucede con las publicaciones sobre historia de la ciencia y las más escasas de historia de la matemática española.

Nosotros, sin embargo, pondremos nuestro foco de interés en la Universidad de Alcalá, y en el marco temporal que se indicará. Una primera razón para ello es que, a pesar de

\footnotetext{
${ }^{1}$ Las restantes, denominadas universidades menores, son, ordenadas cronológicamente, las de Lérida (1300), Murcia (1310), Huesca (1354), Luchente (1423), Barcelona (1430), Gerona (1446), Sigüenza (1472), Zaragoza (1474), Ávila (1482) y Valencia (1500).
} 
ser desde su fundación una de las universidades españolas más importantes ${ }^{2}$, no existen prácticamente trabajos dedicados al estudio de las matemáticas en sus aulas.

Por otra parte, hace tiempo que venimos investigando el estado de la matemática española en el siglo XIX y principios del XX (PERALTA, 1999, 2009a); y, en particular, de la realizada en Madrid (PERALTA, 2000), pues es donde alcanzó su mayor desarrollo. Y en esos estudios se incluía, obviamente, su universidad; aunque Madrid no la tuvo hasta que, en el siglo XIX, se trasladó la de Alcalá a la capital. Queremos, por ello, conocer cuáles fueron los antecedentes de la universidad madrileña, o sea, de la Universidad de Alcalá, en relación con los estudios de matemáticas. Éste será, pues, el fin de nuestra investigación y, por lo ya expuesto, el marco temporal abarcará desde su fundación hasta el término del reinado de Carlos III (en otro trabajo trataremos lo sucedido desde entonces hasta su traslado a Madrid).

Tras las primeras indagaciones, observamos que, salvo en el XVI y quizá principios del XVII, la enseñanza de las matemáticas fue prácticamente inexistente, como también ocurrió, generalmente, en el resto de las universidades españolas; pero mejoró en la segunda mitad del XVIII, participando de los aires ilustrados. Por ello, del período antes indicado nos centraremos, fundamentalmente, en el reinado de Carlos III, pero contemplando también, de algún modo, la etapa que va desde la creación de la Universidad de Alcalá hasta entonces. Concretando, los objetivos de este trabajo son los siguientes:

- Conocer qué presencia tuvieron las matemáticas y cuál fue su nivel de desarrollo en la Universidad de Alcalá durante el reinado de Carlos III. Para ello se plantean también, en particular, estos otros dos objetivos:

- Averiguar qué planes de estudio y reformas se llevaron a cabo en esa etapa.

- Analizar el contenido de los libros recomendados en las asignaturas de matemáticas, para conocer aproximadamente su nivel.

- Saber, a grandes rasgos, cuál era el estado de la educación matemática en la Universidad de Alcalá anteriormente: desde su fundación hasta los tiempos de Carlos III y, en menor medida, en la universidad española en su conjunto.

- Comparar los dos períodos anteriores (antes y durante el reinado de Carlos III).

Se trata, pues, de un trabajo sobre historia de la educación matemática española, enmarcado en las siguientes líneas: 1) Historia de las instituciones y sistemas educativos (KILPATRICK; RICO; SIERRA, 1994); y 2) Evolución de los conceptos matemáticos, su

\footnotetext{
${ }^{2}$ Sobre ella dijo el rey Francisco I de Francia: "Vuestro Cisneros ha logrado realizar más de lo que han hecho varios reyes de Francia” (GUÍA DE LA UNIVERSIDAD COMPLUTENSE (1983-1984), 1983, p. 15).
} 
inclusión en libros de texto antiguos y análisis de sus repercusiones en la enseñanza (GONZÁLEZ; SIERRA, 2003), (PICADO; RICO, 2011).

La estructura del artículo se corresponderá, por tanto, con la que es pertinente en una investigación en historia de la educación matemática (PICADO, 2012).

\section{Fuentes documentales}

Respecto de la heurística de la investigación histórica, esto es, la selección y análisis de fuentes documentales (SALKIND, 1999), para lograr nuestros objetivos, examinaremos:

- Bibliografía correspondiente a la historia de la Universidad de Alcalá.

- Los libros de matemáticas utilizados en Alcalá durante el reinado de Carlos III.

- Estudios sobre la historia de la matemática española, desde el siglo XVI hasta el final del reinado de Carlos III.

- Trabajos sobre historia de la educación y de nuestras universidades en ese período.

Del primero de los asuntos, en especial de su legislación, hemos realizado consultas en el Archivo Histórico de la Universidad Complutense, y analizamos las obras: (AZNAR, 2002) y (SIMÓN, 1992), además de otros trabajos reseñados en la bibliografía. Sobre los textos de matemáticas, utilizados en Alcalá en el reinado de Carlos III, hemos estudiado los libros: (JACQUIER, 1787) y (WOLFIUS, 1763), y añadimos una breve reseña del libro (SEGURA, 1566), pues, aunque muy anterior, puede darnos una idea de su nivel a finales del siglo XVI. Sobre la matemática española nos hemos basado, principalmente, en los trabajos (FERNÁNDEZ VALLÍN, 1893), (LÓPEZ PIÑERO et al., 1983), (PERALTA, 1999, 2009a y 2009b), (REY PASTOR, 1926) y (VICUÑA, 1883) y, para situarla en el marco general de la historia de la ciencia española, en (VERNET, 1975) y (LÓPEZ PIÑERO, 1971). Por último, sobre historia de la educación, hemos recurrido a los textos (VIÑAO, 1982) y (VILLALAÍN, 1997) y, en relación con la Universidad de Salamanca, al libro (PESET; PESET, 1969), además de la legislación recogida en (VILLAGORDO; GARCÍA DE HONORATO, 1771).

\section{Desarrollo de la investigación}

\subsection{La Universidad de Alcalá en sus inicios}

La Universidad creada en Alcalá de Henares, denominada Complutense, la antigua Compluto, fue erigida por el Cardenal Cisneros mediante Bula Pontificia concedida por el 
Papa Alejandro VI. Sus precedentes se encuentran en los Estudios de Alcalá, instituidos en 1460, bajo el reinado de Sancho El Bravo, por Bula Pontificia de Calixto III, en donde posiblemente estudiaría el propio Cisneros (GUÍA DE LA UNIVERSIDAD COMPLUTENSE (1983-84), 1983).

Previamente a la creación de la Complutense, Cisneros había construido el Colegio mayor de San Ildefonso, seis Colegios menores ${ }^{3}$ y un Hospital. Más adelante, erigiría el Colegio Trilingüe, junto a otros Colegios menores, que convirtieron a Alcalá de Henares en una especie de ciudad universitaria. La primera piedra se puso el 14 de marzo de 1499 y la lección inaugural, Las Éticas de Aristóteles, fue pronunciada el 26 de julio de 1508. En el curso 1508-1509 echaría a andar la universidad, con el obispo Pedro del Campo como rector.

Durante la primera mitad del siglo XVI, la universidad alcalaína logró su máximo esplendor, y uno de sus hechos sobresalientes en ese tiempo es la publicación de la Biblia Políglota, dirigida por el propio cardenal, cuya impresión se iniciaría en 1514.

Las universidades funcionaban según el modelo de su fundador y con total independencia unas de otras. La de Alcalá destacó en humanidades, filosofía y teología, aunque no en derecho, pues las Constituciones (estatutos fundacionales) escritas por Cisneros prohibían los estudios de derecho civil en su universidad; y tuvo alguna importancia en el cultivo de las matemáticas. Pero veamos cómo se hallaba, entonces, la matemática española.

\subsection{La matemática española en los siglos XVI-XVII y sus estudios universitarios}

Aunque la ciencia española en el Renacimiento alcanza su plenitud cuando el descubrimiento de América, no sucede lo mismo en matemáticas, cuyos cultivadores no son comparables con los mejores de entonces: los algebristas italianos. Pero, tampoco lo son con los matemáticos portugueses, como Pedro Nunes, quien diría: “... em Espanha ha muy poucos que tenham notícia de Algebra" (NUNES, 1567, p. 14). Y es que en España, en los siglos XVI y XVII, la matemática se dirigía solo a las aplicaciones al cálculo mercantil (la aritmética), y a la navegación, astronomía, técnicas de la guerra... (la geometría). Aparecen, pues, textos únicamente de esas dos ramas, enfocados a distintas profesiones y utilidades: cambios de moneda, arquitectura, agrimensura, sastres y otros oficios; y no se encuentran obras de álgebra, salvo alguna honrosa excepción (en el siglo XVI, Pérez de Moya y Juan de

\footnotetext{
${ }^{3}$ Los Colegios mayores de las Universidades eran centros que impartían enseñanza universitaria de grados mayores (licenciatura o doctorado) y en donde se alojaban los estudiantes. En los Colegios menores se estudiaba el grado menor (bachiller).
} 
Ortega). Y así transcurre a lo largo del siglo, cuando nuestro nivel matemático en sus postrimerías es tan bajo que Felipe II funda, en 1582, una Academia de Matemáticas en Madrid.

Las matemáticas tenían, asimismo, su lugar (auxiliar) en la enseñanza universitaria, figurando en los planes de estudio de las Facultades (menores) de Artes, con una orientación humanística basada en las fuentes clásicas, siguiendo la tradición medieval. El iniciador de esa línea posiblemente fuera Nebrija, que dirigió tres de sus Repetitiones ${ }^{4}$ (1510-1512) a pesos, medidas y números (LÓPEZ PIÑERO, 1971).

En las Facultades de Artes se estudiaban las siete artes liberales: el trivium (gramática, retórica y dialéctica), antecedentes de las Facultades de Filosofía y Letras, y el quadrivium (aritmética, geometría, música y astronomía), germen de las Facultades de Ciencias. A finales del siglo XVI, por ejemplo, en la Universidad de Salamanca, las matemáticas casi se reducían al estudio de los libros de Euclides y a la astronomía de Ptolomeo y Copérnico y sus aplicaciones a cosmografía, gnomónica y náutica, principalmente. Y en la Universidad de Alcalá, se seguía el libro de Juan Segura que aparece en las Referencias (Ioanne Segura, en latín).

Lo hemos consultado, aunque sin analizar detalladamente su contenido; y eso por dos motivos: porque nuestro objetivo principal se centra en la etapa del reinado de Carlos III; pero también porque el libro, escrito en latín, tiene un estilo mucho más compacto que el de los otros dos que se examinarán, pues parece una especie de relato literario (con muy poco espacio dedicado a operaciones, fórmulas, figuras y expresiones matemáticas), y su traducción -aproximada- nos presenta serias dificultades. Digamos unas palabras sobre lo que creemos más reseñable.

La obra, de 157 páginas, tiene seis partes: De Numeris (pp. 13-34), Geometrica Elementa (pp. 35-79), Perspectiva (pp. 80-99), Musica (pp. 100-112), Arithmetica Praxis (pp. 113-135) y Geometrica Praxis (pp. 136-155). En la primera, los números son tratados de una forma casi descriptiva y con una presentación primaria (por ejemplo, un número de varias cifras se escribe separándolas con puntos); se estudian, también, las proporciones y casi nada más, aunque se incluyen, por ejemplo, los números poligonales, siguiendo en ocasiones a los Elementos de Euclides. Esta conexión es mucho más estrecha en la segunda parte, que se ocupa de geometría plana elemental. La Perspectiva es más bien un capítulo sobre Óptica (trata de líneas de reflexión de la luz, pirámides visuales y simetrías especulares, entre otras

\footnotetext{
${ }^{4}$ Como su nombre indica, consistían en repeticiones de sus clases en la Universidad de Salamanca. Hizo nueve, de las que mandó imprimir cinco.
} 
nociones). En Arithmetica Praxis se introducen las operaciones con números naturales (nada de números negativos), figurando como modo alternativo a la multiplicación ordinaria la multiplicación por celosías ${ }^{5}$; y se incluyen la extracción de raíces cuadradas, regla de tres, fracciones y poco más. En Geometrica Praxis se estudian, de modo elemental, polígonos, regiones circulares, prismas, pirámides, conos y demás figuras y cuerpos geométricos; se hallan medidas de longitud, de superficie (que se inician dividiendo la superficie a medir en cuadraditos unidad) y de sólidos; aunque buena parte está destinada a la resolución de problemas de la vida cotidiana, como determinación de la altura de una torre, cálculo de la profundidad de un pozo o volumen de toneles de vino. En fin, de la inclusión de la Música, de la brevedad y nivel del tratado y del análisis realizado, cabe inferir que la matemática que se enseñaba entonces en Alcalá estaba a muy poca altura; similar, por otra parte, a como debería hallarse en la Universidad de Salamanca, a raíz de lo que antes se expuso. En particular, se advierte su dependencia de la geometría griega y sus connotaciones medievales.

En esa línea se manifiesta Rey Pastor, quien afirma que en la segunda mitad del siglo XVI nuestra matemática estaba en franca decadencia, y que no había catedráticos de Matemáticas en las Universidades de Alcalá y de Salamanca (REY PASTOR, 1926).

Volviendo al estado general de la matemática española, se observa que a pesar de los esfuerzos de la Academia de Matemáticas, nuestras obras escritas en los lindes de los siglos XVI y XVII carecen de valor (VERNET, 1975). Así, mientras que a principios del XVII comienza la Revolución Científica en Occidente, que en matemáticas se concreta a lo largo del siglo con el inicio de la matemática moderna, fundamentalmente geometría analítica, cálculo infinitesimal, geometría proyectiva, estadística y cálculo de probabilidades; en España, en cambio, se encuentran en un nivel deplorable, y, en su percepción social, se confunden con la astrología y otras artes esotéricas y de adivinación. Posiblemente en el siglo XVII, tan solo Caramuel estuviera al tanto de los avances que se producían en Europa, pues incluso los tratados de Zaragoza y Omerique se encuentran desfasados con respecto a la geometría de Descartes.

En el XVII las matemáticas, en nuestras universidades, habían llegado casi a desaparecer (LÓPEZ PIÑERO, 1971), y quizá tan solo en Salamanca, y únicamente en las primeras décadas, existiera una cátedra de Matemáticas (en donde prácticamente lo que se estudiaba era geografía) y Astrología (PESET; PESET, 1969). Las universidades españolas siguen su declive, que continuará en el siglo siguiente, y algunos de sus responsables son

\footnotetext{
${ }^{5}$ Este método, debido a los hindúes, fue traído por los árabes en el siglo XIII, y más tarde Luca Pacioli lo recogería en su Summa (1494).
} 
Aristóteles, la escolástica, la gran dependencia de las órdenes religiosas y la Inquisición (que no se relaja hasta mediados del XVIII). Otras causas se encuentran en la estructura de los Colegios mayores (que se irán desmantelando en el último tercio del siglo XVIII), el uso del latín como lengua dominante ${ }^{6}$, el absentismo de los catedráticos, la inexistencia de enseñanza experimental, las irregularidades en la provisión de cátedras, etc. (VERNET, 1975).

En el siglo XVIII se empieza a romper con la línea oficial de la tradición española y la escolástica decadente del XVII. El objetivo principal de las universidades sigue siendo la formación de juristas y teólogos y, en menor medida, de médicos, y hasta su último tercio no existirán universidades con una instrucción adecuada en matemáticas o física (que solo se consideran disciplinas previas para el estudio de la medicina). Sobre ello, y lo sucedido en el XVII, se pronuncia Diego de Torres Villarroel, quien al ganar la cátedra de Matemáticas de la Universidad de Salamanca, en 1726, afirma que había estado sin maestro treinta años y ciento cincuenta sin enseñanza. Igualmente, se hacen patentes esas carencias observando lo que dice un italiano que visita España, en 1755, al referirse a la biblioteca de San Antonio, en Alcalá: “en lugar de Newton, de Descartes, de Galileo, de Malebranche [...], se encuentra a Escoto, Molina, Escobar [...] y otros autores de la misma tela [...]" (VERNET, 1975, p. 136). Sin embargo, como pronto se verá, el panorama va a cambiar en las últimas décadas del siglo. Pero, antes de ello, veamos quiénes fueron los principales matemáticos que enseñaron en Alcalá.

\subsection{Matemáticos de la Universidad de Alcalá}

Entre los mejores matemáticos de estos siglos (FERNÁNDEZ VALLÍN, 1894; LÓPEZ PIÑERO, 1971; LÓPEZ PIÑERO et al., 1983; PERALTA, 1999, 2009a, 2009b; REY PASTOR, 1926; VERNET, 1975; VICUÑA, 1883) se encuentran los siguientes profesores de la Universidad de Alcalá:

Juan Segura (FERNÁNDEZ VALLÍN, 1893). Nació en Cañaveras (Cuenca), estudió y se doctoró en Artes en Alcalá, en donde fue canónigo de la Magistral y catedrático de su universidad en el siglo XVI. Escribió el libro Mathematicae quaedam ..., ya comentado.

Pedro Sánchez Ciruelo (LÓPEZ PIÑERO et al., 1983; PERALTA, 1999; REY PASTOR, 1926; VERNET, 1975; VICUÑA, 1883). Nació en Daroca (Zaragoza) hacia 1470 y

\footnotetext{
${ }^{6}$ No es hasta el 23 de junio de 1768 cuando Carlos III determine la obligatoriedad del castellano en la enseñanza de las primeras letras y escuelas de gramática. Pero en la universidad el latín se mantuvo, todavía, más tiempo (se consideraba más digna la lengua madre que su hija romance): en 1802, el Gobierno ordenó que no se tolerase otra lengua que la latina, y hasta 1813 (!) no se impone definitivamente el castellano.
} 
murió en Salamanca, en 1548. Se licenció en Artes en Salamanca y en Teología y Matemáticas en la Universidad de París. Dio clases en Sigüenza, probablemente en Zaragoza, y en Alcalá durante más de dos décadas. Destaca su Tratactus arithmeticae practice, Cursus quattuor mathematicarum artium liberalium, posiblemente uno de los primeros textos didácticos de matemáticas españoles. Con todo, no fue un matemático renacentista, sino más próximo a Bravardino, Sacrobosto y otros matemáticos medievales. Creó un método aproximado para la extracción de raíces cuadradas y cúbicas y generalizó a cualquier polígono estrellado el teorema de Campano de la suma de los ángulos del pentágono estrellado. Escribió también sobre teología, astronomía, música...

Pedro Esquivel (LÓPEZ PIÑERO et al., 1983; VERNET, 1975). Nacido en Alcalá de Henares hacia 1520 y fallecido hacia 1570, estudió en Alcalá y luego ocuparía su cátedra de Matemáticas. Fue cosmógrafo de Felipe II e iniciador de la geodesia española (es autor de una de las primeras descripciones geodésicas de España: el Atlas de El Escorial).

Diego Pérez de Mesa (LÓPEZ PIÑERO, 1971; REY PASTOR, 1926; ROMERO, 2012). Nació en Ronda (Málaga), en 1563, y falleció en 1632. Estudió Artes y Teología en Salamanca; de 1586 a 1595 fue catedrático de Matemáticas de la Universidad de Alcalá y luego de la de Sevilla. Escribió sobre historia, náutica, cosmología, astrología y matemáticas, especialmente álgebra, destacando su tratado de cosmografía Comentarios de Sphera.

Juan Caramuel Lobkowitz (GARMA, 2000; LÓPEZ PIÑERO et al., 1983; PERALTA, 1999; VERNET, 1975). Nació en Madrid, en 1606, y falleció en Vigevano (Italia), en 1682, en donde fue obispo desde 1673. Estudió en Alcalá (Artes), Salamanca (Teología) y Lovaina (doctorado en Teología) y pasó buena parte de su vida en el extranjero. Dio clases de Teología en Alcalá y otras universidades, y fue matemático, humanista, ingeniero y astrónomo. Mantuvo correspondencia con Descartes y, en sintonía con su Discurso del método, escribió Mathesis Audax, sobre los fundamentos lógicos de las ciencias. También publicó trabajos de física, astronomía y matemáticas. De ésta última, su obra principal es Mathesis Biceps, vetus et nova, en donde expone los conocimientos sobre matemáticas y ciencias entonces existentes. Contiene aportaciones propias sobre sistemas de numeración, un nuevo método aproximado para la trisección del ángulo, un tratamiento más moderno de combinatoria y probabilidades y contribuciones a los logaritmos (de él son las primeras tablas españolas).

Después, durante el reinado de Felipe V, se acentúa la decadencia de la matemática española, en la que ya no existen figuras como Caramuel o Zaragoza. Y, a lo largo de todo el siglo XVIII, como diría Tosca: "Son de España tan forasteras las Matemáticas, que aún entre los eruditos hay pocos que entiendan las voces facultativas más comunes" (REY PASTOR, 
1926, p. 150); y sobre su prácticamente inexistente enseñanza en las universidades en gran parte del siglo, véase lo dicho por Torres Villarroel en el apartado anterior. Así, prácticamente no existen profesores españoles de matemáticas en nuestras universidades reseñables en ese siglo (tan solo hay alguno de su última década, por lo que no será incluido en este trabajo).

Centrándonos ya en los objetivos principales de este estudio, examinaremos en los dos siguientes apartados, respectivamente, cuáles fueron las mejoras impulsadas en la universidad española durante el reinado de Carlos III y, en particular, cómo afectaron a la Universidad de Alcalá.

\subsection{Las reformas}

Carlos III llega a su reinado en España procedente del de Nápoles, con amplia experiencia política. Sabe cuál debe ser su línea de actuación para sacar a España de su atraso: continuar el reformismo de Felipe V y Fernando VI, quienes, ante el grado de analfabetismo imperante, habían iniciado cierta renovación en el ámbito educativo, principalmente con la creación de escuelas y otras instituciones: la Biblioteca Nacional (1711); las Reales Academias de la Lengua (1713), Medicina (1734) e Historia (1738); el Jardín Botánico (1755), etc. Carlos III procede, asimismo, a la modernización y reforma de la enseñanza, tratándola de sustraer de manos de la Iglesia y centralizarla bajo la autoridad del Estado, poniendo el énfasis en la renovación de la educación secundaria y universitaria; y seguirá con la fundación de centros científicos, entre los que se encuentran la Academia de Artillería de Segovia (1763) - institución militar, pero también foco importante de desarrollo de las ciencias -, la Real Academia de Ciencias y Artes de Barcelona (1764) y las Sociedades Económicas de Amigos del País. Pero en sus proyectos chocará con un ambiente adverso fomentado, en cierta medida, por la Iglesia. El primer incidente serio será el motín de Esquilache (1766), a lo que se añadirá el clima enrarecido producido por la expulsión de la Compañía de Jesús (al año siguiente); aunque con sus bienes se crearían importantes instituciones educativas de espíritu liberal.

Durante su monarquía será consciente de la pobre situación en que se encuentran nuestra ciencia ${ }^{7}$ y las universidades; que es extensible a todo el sistema educativo. Por ello impulsará reformas, dando una mayor importancia a las disciplinas científicas y, en general,

\footnotetext{
${ }^{7}$ Ya se han hecho comentarios referentes al estado las matemáticas, que no es muy diferente a lo que sucedía en otras ciencias. Aunque el hecho desencadenante de la polémica sobre la ciencia en España suele considerarse la publicación del artículo Espagne, de Nicolas Masson de Morvilliers, en la Encyclopédie Methodique (1782), en donde llega a afirmarse que nuestro país acaso sea la nación más ignorante de Europa (PERALTA, 2009a).
} 
siguiendo las ideas educativas de los ilustrados (Feijoo, Campomanes y Jovellanos, principalmente).

Sus ideas renovadoras, dirigidas a la educación universitaria y pre-universitaria, se concretan principalmente en las siguientes medidas (VIÑAO, 1982):

a) Reformas en las universidades

El Real y Supremo Consejo de Castilla $^{8}$ decreta reformas generales para todas las universidades, dado que una de las causas de su decadencia es el aislamiento e independencia de cada una. Se inicia, así, el fin de la diversidad y autonomía de las universidades (PESET; PESET, 1969), que se procuran controlar, además, nombrando directores de las mismas, desde 1769, a ministros del Consejo. Las universidades se resistirían a aplicar los cambios y tan solo efectuarían reformas parciales; en todo caso, la nueva legislación lo permitiría en cierto modo, pues no deroga en bloque las Constituciones y Estatutos de cada una de ellas.

Probablemente las principales innovaciones sean el Plan Olavide, de la Universidad de Sevilla (1768) y la Reforma de la Universidad de Salamanca (1771). En el primero, entre otras medidas, en las Facultades de Artes (en donde se imparte el grado de bachiller) se trata de establecer como materias fundamentales, en sus cuatro cursos, matemáticas y física; en concreto, aritmética, álgebra, geometría, trigonometría y física particular (experimental) y general (aristotélica). Pero el plan resultaría fallido al tratar de adaptarlo las demás universidades; así por ejemplo, la de Salamanca estará en contra de la nuevas teorías científicas: "negando que Newton, Gassendi o Descartes puedan entrar en las aulas católicas" (VERNET, 1975, p. 141). En cambio, el Plan de Reforma de la Universidad de Salamanca o Plan Aranda (el conde de Aranda era el presidente del Consejo de Castilla), lo lograría más o menos (en él se basaría el realizado por la Universidad de Alcalá, que pronto comentaremos). La modernización de los planes de estudio, comenzada en Sevilla, continuará en 1771-72 en Salamanca, Alcalá y Valladolid; en Santiago en 1772; Oviedo (1774); Granada (1776)...

El Plan Aranda (VILLAGORDO; GARCÍA DE HONORATO, 1771) da una mayor importancia a las matemáticas, que también se incluyen en las Escuelas de gramática (previas a la educación universitaria, de los que enseguida hablaremos), indicando que sería necesario el estudio de la Sphera, Tablas planetarias y Astrolabio; Geometría y Aritmética; y Cosmografía indiciaria y Perspectiva. Sobre la Facultad de Artes se pronuncia, afirmando que ni la Física experimental ni la Medicina pueden comprenderse sin una base matemática, y propone que la cátedra de Súmulas se subrogue en otra de Geometría, en la que se explique

\footnotetext{
${ }^{8}$ Principal centro de poder y eje de la estructura de gobierno del reino, y su segunda dignidad, tras el rey.
} 
esta materia, junto a Aritmética y Álgebra, que sería optativa, pero obligatoria para alumnos de Medicina, quienes podrían estudiar además otra asignatura (optativa): Matemáticas. El horario lectivo sería de tres horas diarias para cada asignatura (dos por la mañana y una por la tarde).

\section{b) Mayor control de las Escuelas de latinidad y humanidades y Seminarios}

La etapa posterior a los estudios de las primeras letras y previos a la universidad, se cursaba en Escuelas de latinidad, Escuelas de gramática, Colegios de humanidades, Colegios menores, Seminarios eclesiásticos o de nobles y Escuelas profesionales. Tanto la variedad de instituciones como de planes de estudio no permitían precisar exactamente sus funciones ni su correspondencia con los diversos tramos del proceso educativo. En dichos centros, además, no siempre se recibía una buena formación ni su profesorado era el adecuado.

Por todo ello, el Estado ejercerá una mayor jurisdicción sobre esos establecimientos (como la obligación de incluir las asignaturas fundamentales citadas, que determinaba el Plan Olavide para las Facultades de Artes). Se propone, además, reducir su número, impulsando la creación de nuevos centros y la reconversión de muchos que estaban en manos de religiosos (es el preludio de lo que sería la organización de la educación secundaria en el siglo XIX). Entre estos se encuentran los Reales Estudios de San Isidro, el Seminario de Nobles (existente desde su fundación por los jesuitas, en 1725, pero que después de su expulsión ${ }^{9}$ sería regentado por militares y profesores seglares), el Seminario de Vergara, etc. Mención especial merecen los Reales Estudios de San Isidro, procedentes del Colegio de la Compañía de Jesús, establecido en Madrid siglos antes ${ }^{10}$ y que había absorbido la Academia de Matemáticas de Felipe II. Es una de las instituciones con mayor prestigio matemático de la época.

c) Un cierto control y reducción de los centros dirigidos a la carrera religiosa

Continuando con el fin anterior, se tratarán de ordenar las enseñanzas impartidas en los Seminarios conciliares (destinados a la formación de clérigos y sacerdotes). Se prohibirá cursar en ellos enseñanzas de gramática y latinidad, con la consiguiente reducción del número de sus estudiantes (se va diseñando una escuela pública e independiente de la Iglesia).

Veamos, a continuación, cómo van a afectar estos cambios a la Universidad de Alcalá.

\subsection{La situación de la Universidad de Alcalá a partir de las reformas}

\footnotetext{
${ }^{9}$ En el momento de su expulsión regían 188 colegios y 24 seminarios.

${ }^{10}$ Desde principios del XVII se denominaba Colegio Imperial de la Compañía de Jesús, a raíz del apoyo prestado por María de Austria. El Conde de Olivares crearía luego en él unos estudios reales, para mejorar la formación de los futuros dirigentes del país, y le dotaría de cátedras muy bien pagadas, frente a la oposición de las universidades. En 1771, el centro viene a llamarse Reales Estudios de San Isidro (SIMÓN, 1992).
} 
En la Universidad de Alcalá, en 1772, se instaura la Facultad de Leyes, que no había y, como las demás universidades, se reorganiza su plan de estudios. Su Facultad de Artes, en concreto, tendrá siete cátedras: tres de Filosofía eclesiástica, una de Filosofía moral, otra de Física experimental, una de Aritmética, Geometría y Álgebra y otra de Matemáticas. Al acabar sus estudios se podrá acceder al grado de Bachiller en Artes, y serán necesarios para pasar a las Facultades mayores, en donde se opta al grado de Bachiller en la correspondiente Facultad. Un estudiante de una Facultad mayor debía pasar, pues, por la Escuela de primeras letras, la Escuela de gramática, la Facultad de Artes y su propia Facultad.

Nos fijaremos en las dos asignaturas de matemáticas. La de Aritmética, Geometría y Álgebra es de un nivel elemental, y obligatoria para cursar Medicina; mientras que la de Matemáticas, de una mayor complejidad y que se entiende conveniente para el estudio de la medicina y otras ciencias, es voluntaria. Las matemáticas, pues, siguen considerándose solo un instrumento para otras ciencias, pero no como una materia en sí misma, de estructura independiente.

El horario lectivo de las dos asignaturas (AHUCM D-1554) es de dos horas diarias la primera, y tres horas y media diarias la segunda (dos y media por la mañana y una por la tarde). Para la primera se seguirá ${ }^{11}$ el tomo tercero de los cinco que consta Institutiones Philosophicae, de François Jacquier (1711-1788), cuya primera edición, en latín, es de 1757; y, para la asignatura de Matemáticas, los tomos I y III del libro de Christian Wolff (16791754), de cinco volúmenes, publicado en 1710 en alemán, y cuya primera versión en latín, de 1713-1715, se titula Elementa matheseos universae. Ambas obras pronto serán comentadas.

Dejando de momento las Facultades de Artes, los estudios de la Universidad de Alcalá se van deteriorando, y en ello tiene mucho que ver la competencia de otros centros educativos ubicados en la capital. Algunos de los principales son los Reales Estudios de San Isidro - para los que se había establecido un plan de estudios semejante al de las Facultades de Artes y que gozaban de una atención preferente del Gobierno -, el más elitista Seminario de Nobles, el Convento de los Agustinos y el Colegio de San Carlos (VIÑAO, 1982), o los Colegios de mayor antigüedad, de Santo Tomás y de la Encarnación (fundado en 1590).

El caso es que Alcalá tiene cada vez menos alumnos, hasta el punto de que en los estudios de Humanidades o Filosofía generalmente hay tan solo uno o dos matriculados en

\footnotetext{
${ }^{11}$ Ya empieza a esbozarse la cuestión de la procedencia o no de libros de texto, aunque hasta 1813 no se produce la primera referencia oficial sobre ello: texto único, libertad total para el profesor a la hora de elegir o libertad limitada a las obras de una lista elaborada por el Estado (VILLALAÍN, 1997). Los libros que se indican, pues, se refieren a los textos utilizados por la Universidad de Alcalá, pero no con carácter general para las demás.
} 
cada asignatura (RODRÍGUEZ GUERRERO, 2009). Como consecuencia de todo ello se va creando un clima cada vez más favorable al traslado de la Universidad de Alcalá a Madrid.

Volviendo a la finalidad primordial de este artículo, para contemplar mejor cuál era el estado de las matemáticas en Alcalá durante el reinado de Carlos III, como ya se ha indicado, analizaremos, en los dos siguientes apartados, respectivamente, los libros de Jacquier y de Wolff a los que nos referimos anteriormente.

\subsection{Institutiones Philosophicae de François Jacquier}

Hemos consultado el volumen tercero de la edición (JACQUIER, 1787), que consta de 237 páginas (más XV de Introducción e Índice y dos láminas de figuras geométricas), y tiene dos partes: Aritmética y Álgebra (la primera) y Geometría (la segunda). En la primera hay seis capítulos sobre aritmética y álgebra elementales (las cuatro operaciones, números naturales, decimales, fracciones, potencias, cálculo de raíces cuadradas y cúbicas, expresiones algebraicas, proporciones, progresiones y logaritmos) y un apéndice (ecuaciones de primer y segundo grado y sistemas lineales de dos ecuaciones con dos incógnitas sencillos).

La segunda tiene tres secciones: geometría lineal (con seis capítulos y un apéndice), geometría de superficies (tres capítulos) y geometría de sólidos (dos capítulos y un apéndice). En la sección de geometría lineal se realiza un estudio básico de geometría plana (ángulos, polígonos, semejanza, circunferencia, etc.) y medidas de longitud; y, en su apéndice, de trigonometría básica, dirigida a la resolución de triángulos. La geometría de superficies se ocupa de posiciones de puntos, rectas y planos en el espacio, teorema de Pitágoras (aunque no le pone nombre), medidas de superficies de figuras planas, la expresión de $\pi$ (los valores racionales históricos de 22/7 y 113/355 y la forma decimal) y el área del círculo. En geometría de sólidos se estudian, de modo elemental, prismas, pirámides, conos, cilindros y esferas, algunas de sus propiedades y sus áreas y volúmenes; y en su apéndice, titulado líneas curvas, la parábola y la elipse y, solo de pasada, al hablar de secciones cónicas, de la hipérbola.

Posiblemente las cuatro cuestiones más destacadas sean las siguientes. En primer lugar, que no introduce los números negativos en la parte de Aritmética ni opera con ellos, aunque sí opera con expresiones literales (en Álgebra) en donde hay monomios con coeficientes negativos; también aparecen en varias progresiones (como la ...7, 4, 1, -2, -5...), y, aunque solo plantea ecuaciones lineales con soluciones positivas, en la resolución de las de segundo sí introduce el doble signo. En segundo lugar, el tratamiento que da al álgebra (que 
llama arithmetica speciosa ${ }^{12}$ ) en relación con la notación, en donde parece que se dieran los primeros pasos del lenguaje algebraico. Además del signo igual, que posiblemente debido a problemas tipográficos ${ }^{13}$ se representa así: $==$ (pocas veces une los dos signos iguales en uno solo con las rayas más largas), nos referimos, en especial, a la introducción de los paréntesis, que inicialmente escribe con rayas horizontales sobre los elementos afectados; por ejemplo:

$$
\frac{6}{2} \times \overline{4+1}=3 \times 5
$$

En cambio, por causas de nuevo a dificultades tipográficas, escribe

$$
\sqrt{\overline{\mathrm{ax}} \overline{+} \overline{\mathrm{b}}^{2}}=\overline{\mathrm{ax}}+\mathrm{b}
$$

en vez de

$$
\sqrt{\overline{\mathrm{ax}+\mathrm{b}^{2}}}=\overline{\mathrm{ax}}+\mathrm{b}
$$

(sobra la raya sobre $a x$ ). Pero además de estos problemas, sigue quedando en evidencia la imperfecta utilización de los paréntesis, cuando se expresa, por ejemplo (sin paréntesis o la raya encima en los factores del primer miembro): $-a \times+n=-n a$.

La tercera cuestión es que no hay exactamente geometría analítica (no se halla siquiera la ecuación de la recta), aunque se deduce en cambio la ecuación de la parábola $y^{2}=a x-x^{2}$, pero con razonamientos métricos, pasando de puntillas por la noción de coordenadas. La cuarta es que, dentro del nivel elemental del texto, aparecen, sin embargo, varias cuestiones de mayor altura, como la introducción de la serie infinita que resulta al dividir 1 entre 1- $a$ (o sea, $\left.1+a+a^{2}+a^{3}+\ldots\right)$ y alguna otra; los conceptos de curvatura, circunferencia osculatriz..., con razonamientos de tipo infinitesimal, aunque, obviamente, sin el rigor adecuado; etc.

\subsection{Elementa Matheseos Universae de Christian Wolff}

La asignatura de Matemáticas se basa en sus volúmenes I: Qui commentationem de methodo mathematica, aritmeticam, geometricam, trigonometriam planam et analysim, tan finitorum quam infinitorum complectitur y III: Qui opticam, perspectivam, catoptricam, dioptricam, sphaerica et trigonometriam sphaericam atque astronomiam tam sphaericam, quam theoricam complectitur. Como el segundo nos parece de menor interés, pues su parte matemática corresponde únicamente a trigonometría esférica (y astronomía), solo analizaremos el primero, en una reedición (posterior), también en latín (WOLFIUS, 1763).

\footnotetext{
${ }^{12}$ En la sección siguiente se comentará esta denominación y sus connotaciones (nos ha parecido mejor hacerlo así, ya que el libro del que ahora hablamos es posterior al que se estudiará a continuación).

${ }^{13}$ En el siglo anterior, José Zaragoza había tenido que elaborar personalmente los caracteres tipográficos propios del álgebra, entonces inexistentes en las imprentas españolas (PERALTA, 1999).
} 
Ese volumen tiene cuatro bloques: Elementa Arithmeticae, Elementa Geometricae, Elementa Trigonometriae planae y Elementa Analyseos Mathematicae tan finitorum quam infinitorum; que, como puede deducirse, se dedican respectivamente a aritmética, geometría, trigonometría y análisis, aunque con alguna salvedad este último, como indicaremos.

Son 680 páginas en total, además de varias láminas con más de 200 figuras. El primer bloque consta de 10 capítulos; el segundo, que comienza en la página 117, tiene dos partes, geometría plana y geometría del espacio, con 6 capítulos cada una; el tercer bloque empieza en la página 263 y comprende 3 capítulos; y el cuarto va de la página 293 a la 680 y consta de dos partes: análisis finito y análisis infinito, la primera con 2 secciones (con 3 y 8 capítulos, respectivamente), mientras que la segunda contiene 5 (con 3, 6, 2, 3 y 2 capítulos).

Algunas cuestiones que merecen resaltarse son las siguientes: que para aprender a multiplicar se utilice, globalmente, lo que llama abacus pythagoricus (una tabla de 9 filas y 9 columnas, cuya primera fila son los números $1,2, \ldots, 9$, en la segunda están sus dobles, en la tercera sus triples, etc.), en vez de escribir separadamente las tablas del 2, del 3, del 9... (que resultarían considerando la primera y la segunda fila, la primera y la tercera, etc.); la habilidad en la resolución de problemas de ecuaciones y sistemas no lineales, como aquellos de los que solo interesan soluciones enteras: por ejemplo, hallar dos números cuya suma de cuadrados sea igual al cubo del menor (de $x^{2}+y^{2}=y^{3}$, se llega a $x=y \sqrt{y-1}$, que tendrá solución si $y$ - 1, es un cuadrado perfecto: así, $y=5 \Rightarrow x=10, y=17 \Rightarrow x=68$, etc.)...

Pero lo que más nos ha sorprendido es la primera parte, análisis matemático finito, del bloque cuarto, pues en realidad es un tratado de álgebra (vinculado casi siempre a geometría). Parece necesario detenerse en De Arithmetica Speciosa, que es el título del primer capítulo de su primera sección, pues nos recuerda el álgebra de Vieta (1540-1603); lo que no debe extrañarnos demasiado, pues sus escritos no tuvieron verdadera difusión hasta $1646^{14}$ y la primera edición del libro de Wolff data de 1710. Sobre ello hemos de decir, primero, que en este capítulo se introduce muy lentamente la notación algebraica, de acuerdo con su novedad y comprendiendo la dificultad del lector para adaptarse a ella; por ejemplo, indica que $\overline{a+b-c} \times \overline{d-g}$ se expresará $(a+b-c)(d-g)$. Participa de casi las mismas cautelas de Vieta para pasar del álgebra numerosa (con números) al álgebra especiosa (con letras); así, durante el bloque de aritmética no ha tenido reparo en escribir fracciones numéricas con la notación $\frac{3}{4}$, aunque cuando ha sido preciso escribir una con letras la ha denotado $a: b ;$ y no es

${ }^{14}$ La obra de Vieta apareció en 1591 (en Tours, ciudad de segundo orden), cuando su discípulo Anderson ordenó, recopiló y publicó sus escritos, pero no tuvieron auténtica difusión hasta que van Schooten los reeditó, en 1646, en la ciudad de Lyon. 
hasta ahora cuando la escribe $\frac{a}{b}$, diciendo explícitamente que es lo mismo que $a: b^{15}$. También introduce lentamente los números negativos, pues le cuesta desembarazarse de las connotaciones geométricas de la aritmética y el álgebra, a las que vincula exageradamente como Vieta y sus predecesores -; especialmente, le frena el paso al uso meramente algorítmico de las operaciones algebraicas, olvidándose de sus hipotéticas interpretaciones geométricas.

La segunda parte, análisis matemático infinito, de este cuarto bloque es propiamente la correspondiente al análisis, del que hace un estudio bastante completo: cálculo diferencial y sus aplicaciones (tangentes, máximos y mínimos, puntos de inflexión, centros y radios de curvatura...) y cálculo integral, con sus aplicaciones (cuadraturas, rectificación de curvas y cálculo de volúmenes), aunque con una definición imprecisa (normal para esa época) del concepto de diferencial. Quizá lo más reseñable sea la frecuente referencia a curvas históricas (como la concoide de Nicomedes o la espiral de Arquímedes). Aunque las vinculaciones históricas, en realidad, están presentes a lo largo de todo el libro; así, en los logaritmos habla de las aportaciones de Stiefel, Bürgi, Neper...; en el cálculo de $\pi$, de los valores de Ludolf van Ceulen y Vieta, entre otros; en la resolución de ecuaciones cúbicas, de algunos problemas clásicos de la geometría griega; etc.

Con el examen de este libro damos por terminado este estudio, que pasamos, ahora, a resumir y, a continuación, a destacar sus principales conclusiones.

\section{Resumen}

A lo largo de las páginas anteriores se ha hecho una síntesis de la historia de la Universidad de Alcalá - junto a unas breves pinceladas de la universidad española en su conjunto - desde su creación hasta finales del siglo XVIII en relación con la enseñanza de las matemáticas, para lo que ha sido necesario contemplar también, de algún modo, el estado de la matemática - y de la educación en general - en España. Aunque el foco de atención principal se ha puesto en el reinado de Carlos III y sus ideas renovadoras.

Resumidamente, se ha visto lo siguiente:

\footnotetext{
${ }^{15}$ La notación algebraica ha pasado por tres etapas: retórica, cuando a falta de símbolos los cálculos son expresados con palabras (se extiende hasta finales del siglo XV); sincopada, introducida por Pacioli, en la que se emplean abreviaturas (se practica a lo largo del XVI); y simbólica, que utiliza ya signos especiales para datos, incógnitas y operaciones, muy similar a la notación actual (comienza a principios del XVII de la mano de Vieta). En la obra de Nunes (1567) se observan ya rasgos del inicio del álgebra simbólica, al hacer representaciones literales de las cantidades y no vincularse, como hasta entonces, a su consideración como segmentos.
} 
- La Universidad de Alcalá logra su máximo esplendor en el siglo XVI, y las matemáticas también tienen algún relieve. Pero a partir de la segunda mitad, en Alcalá y Salamanca y en toda la universidad española en general, hay pocas cátedras de Matemáticas, y su enseñanza entra en decadencia. Del análisis del libro de Segura se llega, asimismo, a que el nivel matemático es elemental, y alejado del espíritu renacentista de la matemática europea.

- Durante el siglo XVII y a lo largo del casi todo el XVIII, el estudio de las matemáticas prácticamente desaparece de la universidad alcalaína, como igualmente de la mayoría de las universidades españolas.

- La educación, globalmente, y la universitaria en particular, también se encuentra en mal estado, pero Carlos III procura corregir la situación. Para ello se introducen cambios en las escuelas de gramática y universidades, en las que se procura una mayor uniformidad y control por el Estado, y se intenta, de algún modo, que exista una menor influencia de la Iglesia; además, se destaca la importancia de las materias científicas. Las matemáticas, así, vuelven a ocupar su lugar en los planes de estudio.

- Empieza a apreciarse una diferencia en la concepción de las matemáticas. Desde el siglo XVI las matemáticas (aritmética y geometría exclusivamente) están enfocadas al cálculo mercantil y su uso en oficios y - con la astronomía - a sus aplicaciones a la navegación, cosmografía, etc. Desde 1771, en cambio, se consideran asimismo necesarias para el estudio de la física y otras ciencias, aunque todavía no se conciben como ciencia independiente.

- A partir de entonces se siguen las obras reseñadas de Jacquier y Wolff, en latín, cuyas primeras ediciones datan de 1757 y 1710 , respectivamente ${ }^{16}$, y en las que se rompe ya la dependencia de los Elementos de Euclides y la matemática medieval. Se introducen los números negativos, pero con algunas cautelas; y se comienza con el álgebra, aunque con ciertas prevenciones, particularmente en cuanto a su notación. Asimismo, se dan los primeros pasos en la presentación de la matemática moderna, con una leve introducción a la geometría analítica para hallar las ecuaciones de las cónicas, si bien, con razonamientos puramente métricos; se incluyen los logaritmos y, especialmente, se inicia el cálculo infinitesimal.

En el Cuadro 1 se presenta una síntesis de lo anterior:

\footnotetext{
${ }^{16}$ Para completar el estudio de textos: Segura - siglo XVI- y Jacquier y Wolff -siglo XVIII- , hubiéramos querido examinar otro del XVII, pero no ha sido posible, debido a la ausencia casi total del estudio de matemáticas entonces en la universidad alcalaína. Recordemos que Caramuel, uno de los mejores matemáticos españoles del siglo XVII, no dio clases de Matemáticas en Alcalá, sino de Teología (hacia 1630); luego ejercería la docencia en Portugal, Lovaina..., y no volvería a pisar las aulas españolas (GARMA, 2000).
} 


\begin{tabular}{|c|c|c|}
\hline CRONOLOGÍA & ESTADO & CONSIDERACIÓN \\
\hline S. XVI (1 ${ }^{\text {a }}$ mitad $)$ & $\begin{array}{l}\text { La UAH alcanza su máximo esplendor } \\
\text { Las matemáticas tienen algún relieve }\end{array}$ & \multirow{3}{*}{$\begin{array}{l}\text { - Dependencia de la } \\
\text { matemática griega y } \\
\text { medieval. } \\
\text { •Enfocada a mercaderes, } \\
\text { oficios y (junto a la } \\
\text { astronomía) a la navegación, } \\
\text { cosmografía... }\end{array}$} \\
\hline S. XVI ( $2^{\mathrm{a}}$ mitad $)$ & Las matemáticas empiezan su declive & \\
\hline s. XVII y s. XVIII $\left(1^{a} \mathrm{~m}\right.$ & $\begin{array}{l}\text { - Las matemáticas desaparecen de la } \\
\text { UAH. No se dotan cátedras } \\
\text { - Los primeros Borbones inician } \\
\text { tímidas reformas }\end{array}$ & \\
\hline $\begin{array}{l}\text { Reinado de Carlos III (1759-1788) } \\
\text { Reformas en la universidades y } \\
\text { escuelas de gramática: } \\
\text { - Centralización y más poder del } \\
\text { Estado } \\
\text { - Menor influencia de la Iglesia } \\
\text { - Mayor importancia de materias } \\
\text { científicas }\end{array}$ & $\begin{array}{l}\text { - Reformas en la UAH } \\
\text { - Las matemáticas vuelven a los planes } \\
\text { de estudio } \\
\text { - Progresos curriculares. Se introducen: } \\
\text {-Los números negativos } \\
\text {-El álgebra (con cautelas en la } \\
\text { notación) } \\
\text {-Los logaritmos } \\
\text {-La geometría analítica (tímidamente) } \\
\text {-El cálculo infinitesimal }\end{array}$ & $\begin{array}{l}\text {-Rompe su dependencia de } \\
\text { la matemática medieval } \\
\text {-Son necesarias para el } \\
\text { estudio de la física y otras } \\
\text { ciencias } \\
\text {-Se incorporan (con retraso) } \\
\text { al movimiento matemático } \\
\text { europeo } \\
\text {-Todavía no se conciben } \\
\text { como ciencia independiente }\end{array}$ \\
\hline
\end{tabular}

Cuadro 1 - Desarrollo del estado de las matemáticas en la Universidad de Alcalá (UAH)

\section{Conclusiones}

Aunque ya han sido esbozadas de algún modo en el resumen precedente, destaquemos ahora cuáles son, a nuestro juicio, las principales conclusiones a las que se ha llegado en relación con las reformas promovidas por Carlos III:

- Supusieron una mejora en la organización de los estudios previos a la universidad (escuelas de gramática) y en los propios de las universidades, en donde el Estado ejercerá un mayor control, en detrimento del poder ejercido por la Iglesia.

- Dieron más importancia a las materias científicas y, en particular, a la enseñanza de las matemáticas. Esto último se sustanciaría, en la Universidad de Alcalá, en los siguientes aspectos:

1) Después de que, durante algo menos de dos siglos, las matemáticas no figuraran entre las enseñanzas de su universidad o su instrucción tuviera lugar a un nivel muy elemental, por fin van a quedar incorporadas a sus planes de estudio. Concretamente, entre las disciplinas que se imparten en la Facultad de Artes se incluirán dos asignaturas de matemáticas: Aritmética, Geometría y Álgebra, obligatoria para cursar Medicina, y Matemáticas, con contenidos de mayor altura, pero de carácter voluntario para el estudio de la medicina y otras ciencias.

2) Las matemáticas rompen con su excesiva dependencia de las matemáticas griega y medieval, adecuándose, aunque con retraso, a las que corresponden con su tiempo. Se 
presentan, ahora, con un nuevo enfoque: ya no son solo necesarias para el cálculo mercantil y distintos oficios o por sus aplicaciones a la astronomía, la navegación o técnicas de la guerra, sino que también se reconoce su utilidad para el estudio de la física, la medicina y otras ciencias. Con todo, únicamente son consideradas como un instrumento para la ciencia, pero no como una ciencia en sí misma, con su cuerpo de doctrina y su estructura independiente.

3) Coherentemente con el punto anterior, se advierte un importante progreso en los contenidos de matemáticas. Por un lado, se introducen los números negativos, aunque con muchas cautelas. Por otro, se incluye en sus estudios la matemática moderna que había comenzado con la Revolución Científica: el álgebra del Renacimiento, los logaritmos, una incipiente geometría analítica (Descartes) del siglo XVII y el cálculo infinitesimal (Newton y Leibniz) de finales del XVII. A pesar de ello, nuestra matemática se encuentra aún desfasada con Europa, pero al menos se incorpora al importante movimiento que tiene lugar a partir del siglo XVII.

\section{Referencias}

ARCHIVO HISTÓRICO DE LA UNIVERSIDAD COMPLUTENSE DE MADRID (AHUCM), Carpeta D-1554.

AZNAR, R. Cánones y leyes en la Universidad de Alcalá durante el reinado de Carlos III. Madrid: Instituto Antonio de Nebrija / Dykinson, 2002.

FERNÁNDEZ VALLÍN, A. Cultura científica en España en el siglo XVI. Madrid: Real Academia de Ciencias Exactas, Físicas y Naturales, 1893.

GARMA, S. Caramuel y la revolución en las Matemáticas del siglo XVII. In: ESCRIBANO, M. C. (Coord.). Matemáticos madrileños. Madrid: Anaya, 2000, p. 105-140.

GONZÁLEZ, M. T; SIERRA, M. El método de investigación histórica en la didáctica del análisis matemático. In: CASTRO, E. (Coord.). Investigación en Educación Matemática, SIMPOSIO DE LA SOCIEDAD ESPAÑOLA DE INVESTIGACIÓN EN EDUCACIÓN MATEMÁTICA, 7, 2003. Granada: Universidad de Granada, 2003, p. 109-130.

GUÍA DE LA UNIVERSIDAD COMPLUTENSE (1983-84). Madrid: Ed. de la Universidad Complutense, 1983.

JACQUIER, F. Institutiones philosophicae, Tomus III. Madrid: Ex Officina Ildephonsi à Lopez, 1787.

KILPATRICK, J.; RICO, L.; SIERRA, M. Educación Matemática e Investigación. Madrid: Síntesis, 1994.

LÓPEZ PIÑERO, J. M. Ciencia y técnica en la sociedad española de los siglos XVI y XVII. Barcelona: Labor, 1971. 
LÓPEZ PIÑERO, J. M; GLICK, T. F.; NAVARRO, V.; PORTELA, E. Diccionario histórico de la ciencia moderna en España. Barcelona: Península, 1983.

NUNES, P. Libro de Algebra en Arithmetica y Geometria. Amberes: Casa da la Biuda y herederos de Iuan Stelsio, 1567.

PERALTA, J. La matemática española y la crisis de finales del siglo XIX. Madrid: Nivola, 1999.

PERALTA, J. La Matemática madrileña en el panorama español de 1800 a 1936. In: ESCRIBANO, M. C. (Coord.). Matemáticos madrileños. Madrid: Anaya, 2000, p. 183-230.

PERALTA, J. La matemática española del siglo XIX. In: CONSEJERÍA DE EDUCACIÓN, UNIVERSIDADES, CULTURA Y DEPORTES. FUNDACIÓN OROTOVA DE HISTORIA DE LA CIENCIA (Coord.). La Ciencia antes de la Gran Guerra. Actas Año XVII. Encuentros Educativos. Canarias: Imp. Reyes, 2009a, p. 211-236.

PERALTA, J. Una aproximación a las Matemáticas y a la comunidad científica en la España Ilustrada. Cátedra Nova, Badajoz, n. 28, p. 145-165, jun. - dic. 2009 b.

PESET, M.; PESET, J. L. El reformismo de Carlos III y la Universidad de Salamanca. Salamanca: Ed. Universidad de Salamanca, 1969.

PICADO, M. El Sistema Métrico Decimal en libros de texto de matemáticas en España durante la segunda mitad del siglo XIX (1849-1892). 2012, 428 p. Tesis (Doctorado en Didáctica de la Matemática). Departamento de Didáctica de la Matemática, Universidad de Granada, Granada, 2012.

PICADO, M.; RICO, L. La selección de textos en una investigación histórica en Educación Matemática. Épsilon, Puerto Real, v. 28 (1), n. 77, p. 99-112, abr. 2011.

REY PASTOR, J. Los matemáticos españoles del siglo XVI. Madrid: Biblioteca Sciencia, 1926.

RODRÍGUEZ GUERRERO, C. El Instituto del Cardenal Cisneros de Madrid (1845-1877). Madrid: Consejo Superior de Investigaciones Científicas, Biblioteca de Historia, 2009.

ROMERO, F. Algebraic symbolism in the first algebraic works in the Iberian Peninsula. Philosophica, Gante, v. 87, n. 4, p. 117-152, 2012.

SALKIND, N. J. (1999). Métodos de investigación. México: Prentice Hall, 1999.

SEGURA, I. Mathematicae quaedam selectae propositiones, ex Euclides, Boëtij, \& et antiquorum aliorum libris decerptae, quibus literales disciplinae in compedium quoddam redactae non magno negotio peruiae addifcentibus erum. Alcalá de Henares: Andreas de Angulo, 1566.

SIMÓN, J. Historia del Colegio Imperial de Madrid. Madrid: Instituto de Estudios Madrileños / Dorsa, 1992.

VERNET, J. Historia de la ciencia española. Madrid: Instituto de España, Cátedra Alfonso X el Sabio, 1975.

VICUÑA, G. Los matemáticos del siglo XVII. Revista contemporánea, Madrid, año IX, t. XLVI, v. I, p. 5-21, jul. 1883.

VILlAGORDO, A.; GARCÍA DE HONORATO, T. Segundo tomo de la Coleccion de Reales Decretos, Ordenes y Cedulas de su Magestad (que Dios guarde) de las Reales provisiones y Cartas-Ordenes del Real y Supremo Consejo de Castilla, dirigidas à esta Universidad de 
Salamanca, para su govierno, que siguen desde el mes de Julio del año pasado de 1770 hasta el mes de Noviembre del presente año de 1771. Salamanca: Eugenio García de Honorato y Nicolás Villagordo, 1771.

VILLALAÍN, J. L. Manuales escolares en España, Tomo I: Legislación (1812-1939). Madrid: Universidad Nacional de Educación a Distancia, 1997.

VIÑAO, A. Política y educación en los orígenes de la España Contemporánea. Examen especial de sus relaciones con la enseñanza secundaria. Madrid: Siglo XXI, 1982.

WOLFIUS, C. Elementa matheseos universae, Tomus I y III. Genevae: Henricum-Albertum Gosse \& Socios, 1763.

Submetido em Maio de 2015. Aprovado em Novembro de 2015. 